\title{
Dislocations and melting in two and three dimensions
}

\author{
Tallon, Jeffery L.
}

Published in:

Physical Review B

Link to article, DOI:

10.1103/PhysRevB.22.453

Publication date:

1980

Document Version

Publisher's PDF, also known as Version of record

Link back to DTU Orbit

Citation (APA):

Tallon, J. L. (1980). Dislocations and melting in two and three dimensions. Physical Review B, 22(1), 453-455. https://doi.org/10.1103/PhysRevB.22.453

\section{General rights}

Copyright and moral rights for the publications made accessible in the public portal are retained by the authors and/or other copyright owners and it is a condition of accessing publications that users recognise and abide by the legal requirements associated with these rights.

- Users may download and print one copy of any publication from the public portal for the purpose of private study or research.

- You may not further distribute the material or use it for any profit-making activity or commercial gain

- You may freely distribute the URL identifying the publication in the public portal

If you believe that this document breaches copyright please contact us providing details, and we will remove access to the work immediately and investigate your claim. 


\title{
Comments and Addenda
}

The section Comments and Addenda is for short communications which are not appropriate for regular articles. It includes only the following types of communications: (1) Comments on papers previously published in The Physical Review or Physical Review. Letters. (2) Addenda to papers previously published in The Physical Review or Physical Review Letters, in which the additional information can be presented without the need for writing a complete article. Manuscripts intended for this section must be accompanied bv a bricf abstract for information-retrieval purposes. Accepted mamuscripts follow the same publication schedule as articles in this journal. and page proofs are sent to authors.

\section{Dislocations and melting in two and three dimensions}

\author{
Jeffery . L. Tallon* \\ Department of Structural Properties of Materials, The Technical University of Denmark, \\ Building 307, DK-2800 Lyngby, Denmark \\ (Received 7 May 1979)
}

\begin{abstract}
Comments are presented on the recent theories of two-dimensional melting which envisage melting as proceeding via two second-order transitions comprising dislocation dipole dissociation followed by disclination dipole dissociation. It is suggested that if the configurational entropy is properly included, the model system may jump discontinuously from a volume below the dislocation transition to a volume above the disclination transition so that both transitions are virtual and are hidden in the first-order discontinuity. A reinterpretation of the recent moleculardynamics simulation of two-dimensional melting of Frenkel and McTague, reveals that such is the case for a Lennard-Jones system. There may be no fundamental difference between twoand three-dimensional melting.
\end{abstract}

Recently, Nelson, ${ }^{1}$ Halperin and Nelson, ${ }^{2}$ Nelson and Halperin, ${ }^{3}$ and Young ${ }^{4}$ have investigated quite extensively the consequences of a dislocation theory of second-order two-dimensional (2D) melting, modeling their approach on that of Kosterlitz and Thouless, ${ }^{5}$ which proposes that the melting is mediated by dissociation of dislocation dipoles. This specific mechanism was in fact observed earlier by Cotterill and Pedersen ${ }^{6}$ in molecular dynamics simulated twodimensional melting of a Lennard-Jones system, and the essential features of the Halperin-Nelson theory are apparently confirmed by the recent molecular dynamics calculations of Frenkel and McTague ${ }^{7}$ (FM). Specifically, Halperin and Nelson (HN) have suggested that if melting in 2D is not first order, then it is a two-step process involving a transition to a "hexatic" phase driven by dislocation dipole dissociation, followed by a transition to an isotropic phase at higher temperature which is driven by disclination dipole dissociation. The purpose of the present article is to draw attention to a number of inadequacies in the current formulation of the dislocation theory, but to do so within the context of a viewpoint which is sympathetic towards the dislocation model. In particular, it is shown that, contrary to the conclusions of Frenkel and McTague, melting in 2D is probably first order as it is in three-dimensions (3D) and that the two-step melting process postulated by Halperin and Nelson occurs in the virtual region between the melting line and the freezing line.

Firstly, some comments on the historical background would appear to be in order. The notion of dislocation mediated phase transitions has rather diffuse origins, and even before explicit dislocation theories of melting were discussed, Frank and van der Merwe ${ }^{8}$ studied the role of dislocations in the structure of a monolayer on a substrate. Detailed dislocation theories of melting have been presented by Rothstein, ${ }^{9}$ Mizushima, ${ }^{10}$ Ookawa, ${ }^{11}$ and many others, and the notion of dislocation dipole dissociation was probably first introduced by KuhlmannWilsdorf. ${ }^{12}$ Although each was moderately successful in characterizing melting parameters, the dislocation model was often regarded with skepticism. ${ }^{13,14}$ The credibility of the dislocation model was greatly enhanced, however, by Cotterill and Pedersen's observation of dipole dissociation during melting. ${ }^{6}$ This work advances the status of the dislocation from being a hypothetical paradigm for the disorder associated with the liquid state, to being an established participant in the melting process, if not necessarily in the final liquid state. Moreover, the role of dislocations in melting is not confined to systems of two dimensions only, but has also been established for three- 
dimensional systems. ${ }^{15}$ There appears from this work to be no fundamental difference between the melting of 2D and 3D systems. It is interesting also to note that the essential difference between a 2D lattice and a 3D lattice is that, in the former, the longwavelength phonons destroy the conventional longrange order. In the computer simulation of FM these long-wavelength phonons are absent and yet the two second-order transitions predicted by $\mathrm{HN}$ to constitute 2D melting, are confirmed. It is likely, then, that these transitions are not directly related to this special characteristic of 2D lattices and may, in fact, be common to both $2 \mathrm{D}$ and $3 \mathrm{D}$ lattices.

The central inadequacy of the $\mathrm{HN}$ theory is that it fails to consider explicitly the entropy of the system. This is offset in part by the fact that the strain energy of a dislocation dipole is not an internal energy as stated by Kosterlitz and Thouless ${ }^{5}$ but it is a Helmholtz function. ${ }^{16}$ The vibrational entropy is therefore implicitly contained in the strain energy which happens to be used by $\mathrm{HN}$ in a manner consistent with a Helmholtz function. There are, however, important configurational contributions to the system entropy which are omitted. For a large number of materials there is a volume-independent entropy change on melting of $R \ln 2,{ }^{17-19}$ and for some materials all or part of the communal entropy, $R$ appears on melting. ${ }^{20}$ In the two-dimensional model system, the advent of the latter entropy is probably unlikely, but in actual monolayer systems in which one could expect greatly enhanced diffusivities (akin to the high surface mobilities for solids ${ }^{21}$ ) the communal entropy could be significant.

One is thus presented with a situation analagous to that which occurs in the modified Born instability criterion for melting. ${ }^{22-24}$ The original Born criterion ${ }^{25}$ for melting leads to a second-order melting transition, but when considerations of the configurational, volume-independent entropy are incorporated into the criterion the transition becomes first order. In the present context, we may say that the appearance of the configurational entropy at the disclination unbinding transition allows the system to transform discontinuously from a lower volume which may even be lower than the volume at which the dislocation unbinding occurs. It is therefore possible that melting, whether in 2D or 3D, is a discontinuous transition from below a virtual dislocation unbinding transition to above a virtual disclination unbinding, and under normal experimental conditions neither of these hidden transitions would be seen.

There are at least two ways of realizing the hidden transitions. One is to clamp the area or volume so as to prevent the discontinuity and essentially superheat the lattice. The other is rather less artificial. When it is said that melting is first order, this only refers to the relative properties of the ideally infinite bulk phases and locally, on the finite atomic level, the transition is not abrupt. In particular, one might expect to find these two transitions separately realized in the solid-liquid $(s-1)$ interface (which in a three-dimensional Lennard-Jones system is as wide as eight atom diameters ${ }^{26,27}$ ). On the ordered side of the interface, it seems certain that dislocation dipole generation will be evident, and the structure there will be anisotropic, while at the disordered side of the interface, one may find the proposed disclination dissociation effectively completing the transition to isotropy. Computer simulations of the $s-1$ interface have been reported, ${ }^{26,27}$ but the analysis of these was confined to average structures and not specific instantaneous structures.

The other method of realizing the hidden transitions, namely, that of superheating by fixing the area or volume, has in fact been used by FM. They carried out isochoric heating and cooling runs on a Lennard-Jones system at a reduced density of $\rho^{*}=0.8$. Two transitions, qualitatively akin to those predicted by $\mathrm{HN}$, were located, one at a reduced temperature of $T^{*} \simeq 0.36$ and reduced pressure of $P^{*} \simeq-0.2$, and the other at $T^{*} \simeq 0.57$ and $P^{*}=1.4$. These two sets of points are just two separate points on the first-order melting line located by Toxvaerd. ${ }^{28}$ The conclusion of FM that their melting transition is higher than first order is therefore untenable. Their results are, rather, consistent with superheating and supercooling along a continuous path between solid and liquid. In fact the two $\left(P^{*}, \rho^{*}, T^{*}\right)$ points lie within the melting domain. Figure 1 shows the $\left(\rho^{*}, T^{*}\right)$ melting and freezing curves found by Toxvaerd ${ }^{28}$ and these bracket the transition points of FM.

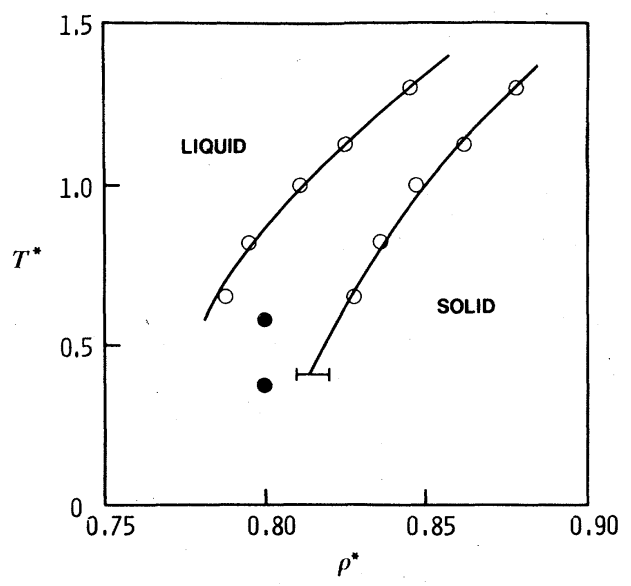

FIG. 1. Temperatures and densities along the melting and freezing lines reported by Toxvaerd for a 2D Lennard-Jones condensed system. Data points are denoted by open circles. The solid points are the transition points located by Frenkel and McTague, and both lie entirely within the first-order melting domain. 
The absence of hysteresis on heating and cooling would appear to confirm that the melting domain is not a heterophase region.

These two results of FM and Toxvaerd, ${ }^{28}$ when taken together, give strong support to the view expressed above, that melting in $2 \mathrm{D}$ and $3 \mathrm{D}$ is a firstorder transition around separate virtual dislocation and disclination unbinding transitions. The principal difference between 2D and 3D first-order melting would probably lie in the relative widths and separation of the hidden transitions, each of these being larger in 2D due to the geometrical constraint. Further support for the first-order character of $2 \mathrm{D}$ melting may be found in at least two other computer simulations ${ }^{29,30}$ and in the fact that successive surface layers of a $3 \mathrm{D}$ solid melt abruptly at progressively higher temperatures, bounded by the bulk melting point. $^{31}$ The outermost layer is, of course, a commensurate monolayer.

A proper formulation of such a transition around two hidden transitions presents a formidable task. Computer simulations of $2 \mathrm{D}$ and $3 \mathrm{D}$ liquids suggest that if the structure were to be resolved into distributions of dislocation dipoles the dipole separation would never be large compared to a Burgers vector. The effect of bringing two dislocations together within a few Burgers vectors of each other is that the core structure alters ${ }^{32}$ and in the case of a dipole, the cores will dilate with decreasing width. Thus, in the regime of interest, the core energy is by no means constant and the simple logarithmic energy function upon which the HN formulation is based is untenable. It is evident, however, that there are certain systems for which the HN formulation is valid. Moreover, there may be some systems for which the discontinuous volume jump occurs near or above the dislocation unbinding transition. In this respect it may be recalled that certain lattices display premelting effects. $^{33}$

\section{ACKNOWLEDGMENTS}

The author is grateful to R. M. J. Cotterill and J. U. Madsen for many interesting discussions concerning this work which was financially supported by the New Zealand Department of Scientific and Industrial Research.
"Permanent address: Physics and Engineering Laboratory, Department of Scientific and Industrial Research, Private Bag, Lower Hutt, New Zealand.

${ }^{1}$ D. R. Nelson, Phys. Rev. B 18,2318 (1978).

${ }^{2}$ B. I. Halperin and D. R. Nelson, Phys. Rev. Lett. 41, 121 (1978).

${ }^{3}$ D. R. Nelson and B. I. Halperin, Phys. Rev. B $\underline{19}, 2457$ (1979).

${ }^{4}$ A. P. Young, Phys. Rev. B 19 , 1855 (1979).

5. M. Kosterlitz and D. J. Thouless, J. Phys. C 6, 118 (1973).

${ }^{6}$ R. M. J. Cotterill and L. B. Pedersen, Solid State Commun. 10, 439 (1972).

${ }^{7}$ D. Frenkel and J. P. McTague, Phys. Rev. Lett. 42,1632 (1979).

${ }^{8}$ F. C. Frank and J. H. van der Merwe, Proc. R. Soc. London Ser. A 198, 205 (1949).

9J. Rothstein, J. Chem. Phys. 23, 218 (1955).

${ }^{10}$ S. Mizushima, J. Phys. Soc. Jpn. 15, 70 (1960).

${ }^{11}$ A. Ookawa, J. Phys. Soc. Jpn. 15, 2191 (1960).

${ }^{12}$ D. Kuhlmann-Wilsdorf, Phys. Rev. A 140,1599 (1965).

${ }^{13}$ L. V. Woodcock, Nature London Phys. Sci. 232, 63 (1971).

${ }^{14} \mathrm{~F}$. C. Frank and J. W. Steeds, in The Physics of Metals 2, edited by P. B. Hirsch (Cambridge University Press, Cambridge, England, 1975), pp. 68,81.

${ }^{15}$ R. M. J. Cotterill, W. Damgaard Kristensen, and E. J. Jensen, Philos. Mag. 30, 245 (1974).

${ }^{16}$ D. C. Wallace, in Solid State Physics, edited by H. Ehrenreich, F. Seitz, and D. Turnbull (Academic, New York, 1970), Vol. 25 , p. 301.
${ }^{17}$ S. M. Stishov, I. N. Makarenko, V. A. Ivanov, and A. M. Nikolaenko, Phys. Lett. A 45, 18 (1973).

${ }^{18}$ M. Lasocka, Phys. Lett. A 51, 137 (1975).

${ }^{19}$ J. L. Tallon, Phys. Lett. A $\underline{76}, 139$ (1980).

${ }^{20}$ L. V. Woodcock and K. Singer, Trans. Faraday Soc. 67, 12 (1971)

${ }^{21}$ J. L. Tallon, W. H. Robinson, and S. I. Smedley, J. Phys. C 10, L579 (1977).

22J. L. Tallon, W. H. Robinson, and S. I. Smedley, Nature (London) 266, 337 (1979).

${ }^{23}$ J. L. Tallon, W. H. Robinson, and S. I. Smedley, Philos. Mag. 36, 741 (1977).

24J. L. Tallon, Philos. Mag. A $\underline{39}, 151$ (1979).

${ }^{25}$ M. Born, J. Chem. Phys. 7, 591 (1939).

${ }^{26}$ A. J. C. Ladd and L. V. Woodcock, J. Phys. C 11,3565 (1978).

${ }^{27}$ S. Toxvaerd and E. Praestgaard, J. Chem. Phys. $\underline{67}, 5291$ (1977).

${ }^{28}$ S. Toxvaerd, J. Chem. Phys. 69, 4750 (1978).

${ }^{29}$ R. W. Hockney and S. P. Goel, Chem. Phys. Lett. 35, 500 (1975).

${ }^{30}$ F. Tsien and J. P. Valleau, Mol. Phys. 27, 177 (1974).

${ }^{31}$ J. Q. Broughton and L. V. Woodcock, J. Phys. C $\underline{11}, 2743$ (1978).

${ }^{32}$ F. R. N. Nabarro, Theory of Crystal Dislocations (Clarendon, Oxford, 1967), p. 143.

${ }^{33}$ A. R. Ubtelohde, The Molten State of Matter (Wiley, Chichester, 1979). 